\title{
DESARROLLO E IMPLEMENTACIÓN DE FOTOCONTROL TEMPORIZADO CON TECNOLOGÍA DE BAJO CONSUMO
}

Fredy Hernán Martínez

Magister en Automatización y Control Docente planta de la Universidad Distrital Francisco José de Caldas

fhmartinezs@udistrital.edu.co

Bogotá, Colombia

\section{Edward Jacinto Gómez}

Ingeniero en Control Electrónico e Instrumentación

Docente planta de la Universidad Distrital Francisco José de Caldas ejacintog@udistrital.edu.co

Bogotá, Colombia

\section{David Ramírez Buitrago}

Tecnólogo en Electrónica

Universidad Distrital Francisco José de Caldas

david_richardsb@hotmal.com

Bogotá, Colombia

Tipo: Artículo reporte de caso

Fecha de Recepción: Julio 1 de 2013

Fecha de Aceptación: Agosto 6 de 2013

\section{DEVELOPMENT AND IMPLEMENTATION OF TIMED PHOTO CONTROL USING TECHNOLOGY OF LOW CONSUMPTION}

\begin{abstract}
This paper presents the development and implementation of a photo control with low consumption technology equipped with a microcontroller, which controls tension, luminosity and synchronization variables with the network by using an output with a PWM mode to produce a Vdc output of on the power relay. The microcontroller performs a voltage analogue sampling over the sine wave and a moving average filter is made to eliminate the noise of the wave.
\end{abstract}

The timing takes place when the power relay contact is closed and the time to be timed is programmed by the user as it is required, the photo control presents an ultra low power mode on the microcontroller with a supercap.

Key words: digital filter, microcontroller, pulse width modulation, timing

\section{RESUMEN}

En este trabajo se presenta el desarrollo e implementación de un fotocontrol con tecnología de bajo consumo desarrollado con un microcontrolador, el cual controla variables de tensión, luminosidad y sincronización con la red, por medio de una salida con modo PWM producir una salida de Vdc en el relé. El microcontrolador realiza un muestreo análogo de voltaje sobre la onda sinusoidal y se hace un filtro de promedio móvil para eliminar ruido de la onda.

La temporización se realiza cuando el contacto del relé está cerrado y el tiempo a temporizar es programado por el usuario el cual lo requiera, el fotocontrol presenta un modo de ultra bajo consumo en el microcontrolador con un supercap.

Palabras claves: filtro digital, microcontrolador, modulación por ancho de pulso, temporización. 


\section{INTRODUCCIÓN}

Este proyecto es un artículo de investigación científica y tecnológica el cual fue desarrollado entre el grupo de investigación IGITI y la empresa Inadisa S.A. la cual financio el proyecto el cual se describe a continuación.

El fotocontrol es un sistema que está encargado de encender y apagar las luminarias de alumbrado público, las cuales están compuestas por tres elementos los cuales son arrancador, balasto y la bombilla de sodio y sus márgenes de operación están regidos bajo la norma NTC2470[1].

En Colombia cada poste de alumbrado público tiene su elemento de control, denominado fotocontrol, pero el consumo de potencia en la luminaria es alto, al estar activada de forma innecesaria en algunos puntos de la red. De acuerdo a las condiciones de la red de alumbrado público, se debe garantizar el encendido de la luminaria, a lo largo de la vida útil. En este mismo sentido el circuito de control debe operar dentro de los márgenes de consumo de potencia exigidos por la norma.

El desarrollo consiste en realizar un fotocontrol temporizado el cual desactive la carga en algunas horas de la noche, este se desarrollo con el microcontrolador MSP430F2002 el cual se encarga de procesar las variables de voltaje e iluminación para controlar los contactos de un relé electromecánico y tener unos rangos de funcionamiento entre 105 Vac y 305 Vac y procesar los datos de voltaje y hacer funcionar el relé a unos rangos de $20 \mathrm{Vdc}$ a $24 \mathrm{Vdc}$ por medio de una salida PWM del microcontrolador haciendo una temporización nocturna definida por el usuario y presentando modo de ultra bajo consumo al momento de ausencia de tensión en la red para que no pierda el conteo nocturno en la temporización [2], [3], [4].

Este desarrollo busca mejorar las características de los fotocontroles anteriormente hechos en la empresa INADISA S.A de forma análoga y, solucionar problemas de consumo de potencia, a un costo del producto razonable [5], [6].
En Uruguay se desarrollan fotocontroles en la empresa IMTRAN los cuales tienen rangos de tensión de funcionamiento de 105 Vac a 305 Vac con protecciones contra sobretensiones de la red con un modo de funcionamiento de Fail On.

En el fotocontrol desarrollado se implementaron algunas mejoras adicionales en cuanto a la reducción del ruido del relé y de la corriente transitoria, al disponer de un microcontrolador el cual conmutar la carga cerca del cruce por cero de la onda sinusoidal rectificada y permite que al momento de hacer conmutar la carga que la corriente transitoria no sea tan elevada y no genere un fenómeno en el relé de micro soldar el contacto y se deteriore el producto [7], [8], [9].

\section{DESARROLLO}

El fotocontrol temporizado con modos de bajo consumo desarrollado en INADISA S.A, conmuta la carga por medio de un relé electromecánico con un modo de funcionamiento Fail On, cuando esta de día el contacto del relé está abierto y mantiene la luminaria apagada y cuando esta de noche cierra el contacto del relé $\mathrm{y}$ activa la luminaria.

El fotocontrol tiene un microcontrolador para el control de las variables de luz, voltaje y sincronización con la red de $60(\mathrm{~Hz})$ y realizar la salida con modos de PWM para el control del relé, el microcontrolador que se escogió para realizar la aplicación fue un MSP430F2002 de Texas Instruments por que presenta 5 modos de ultra bajo consumo y el precio de este microcontrolador es bajo.

Para poder programar el microcontrolador de la familia MSP430 es necesario tener el siguiente software Code Composer Studio (CCS) o (IAR) Wokbench los cuales se pueden descargar de forma gratuita de la pagina de Texas Instruments, para CCS se permite 16KB de código escrito en lenguaje $\mathrm{C}$.

Diagrama de flujo de las partes desarrolladas en el proyecto se muestra en la figura 1 . 


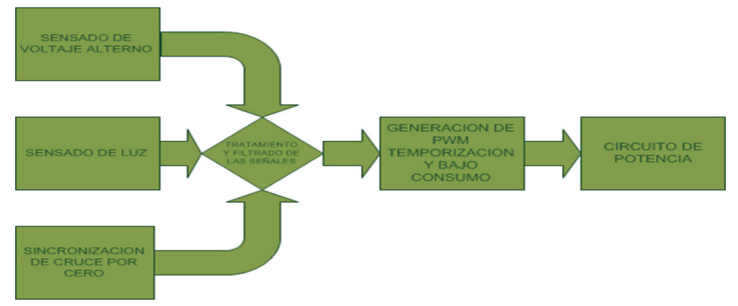

Figura 1. Diagrama de flujo de las partes desarrolladas en el proyecto

\subsection{Sistema de rectificado de onda completa y acondicionamiento de las señales}

A la señal de entrada sinusoidal se le realizo un rectificado de onda completa el cual debe ser capaz de soportar tensiones de 105Vac a $305 \mathrm{Vac}$ con corrientes de $1 \mathrm{~A}$ a $5 \mathrm{~A}$ a $60 \mathrm{~Hz}$, para lo cual se utilizaron los diodos rectificadores MIC 4007 que tienen estas características de funcionamiento. Para el cálculo del valor Root Mean Square (RMS) de una onda seno se tiene la ecuación (1) la cual se evalúa en intervalos los cuales son t1 y t2 dando un número.

$$
\int_{t 1}^{t 2} \sin (t) \cdot d t
$$

Luego del sistema de rectificado se reduce la corriente y el voltaje de entrada al circuito, con una fuente resistiva y un diodo zener de $3,3 \mathrm{~V}$ directo (Vdc), acondicionando la señal de salida a 3,3 (Vdc) que está dentro del rango de alimentación del microcontrolador MSP430F2002 [10].

De la salida rectificada se hace un detector de cruce por cero el cual está compuesto por dos resistencias las cuales bajan la tensión de la salida del rectificador y un diodo zener de 3,3 (Vdc) que limita el voltaje, para que posteriormente sea tratada en el microcontrolador.

Se realiza un sensor de voltaje compuesto por dos resistencias en serie para bajar la tensión de salida rectificada, y detectar las variaciones de tensión, estas resistencias se calculan mediante la fórmula de un divisor de voltaje ecuación (2) para obtener, frente a los rangos de en- trada de 105 (Vac) a 305 (Vac) en la red, una salida entre 0 y 3,3 (Vac) para que los rangos de voltaje del sensor puedan ser leídos por las entradas análogas del microcontrolador [11].

$$
\text { Vout }=\frac{\operatorname{Vin} \cdot R 2}{R 1+R 2}
$$

Se hace un sensor de luz con un fototransistor y una resistencia, calibrado por un luxómetro para dar a la salida una tensión según el nivel de luminosidad del ambiente regida por la norma NTC2470.

\subsection{Tratamiento de las señales}

El tratamiento de las señales se realiza a través de un microcontrolador MSP430F2002 de Texas Instruments, la primera señal a tratar es la detección del cruce por cero para sincronizar la salida con los modos de PWM del microcontrolador [12].

Esta detección se hace a través de una interrupción por puerto, el microcontrolador MSP430F2002 posee estas interrupciones por todos los pines los cuales son configurables por software.

Posteriormente se hacen las lecturas análogas de los sensores de luz y voltaje en el microcontrolador, y se realiza la conversión del valor análogo a un valor digital que está dentro del rango de 0 a 1023, ya que la resolución del conversor es de 10 bits y una velocidad de muestreo de 200 MSPS [11].

Para el sensor de voltaje se toman 20 muestras por ciclo, el ciclo de la onda seno del sensor de voltaje es de $8.33 \mathrm{~ms}$ por lo cual entre cada muestra debe haber un retardo de $416.5 \mathrm{uS}$, se realizo un filtrado de promedio móvil a las muestras, para eliminar el ruido de la onda rectificada de entrada al microcontrolador ecuación (3). Tomando el valor máximo después de realizar las 20 muestras y dividiendo ese valor entre 20 ecuación (4).

$$
\text { Filtro Voltaje }=\frac{1023 \cdot \text { Vadc }}{20}
$$




$$
\begin{aligned}
& \text { Pulse Low }=\frac{8,33[\mathrm{mS}]}{20} \\
& \text { Pulse Low }=416,5[\mu \mathrm{S}]
\end{aligned}
$$

Para el sensor de luz se toman 5 muestras después de hacer el muestreo y filtrado del sensor de luz, y se realiza un filtro de promedio móvil para tomar un valor más exacto de la lectura del sensor ecuación (5).

$$
\text { Filtro Luz }=\frac{1023 \cdot \operatorname{Vadc}}{5}
$$

En la noche se puede presentar fenómenos tales como rayos para el cual el fotocontrol debe tener un tiempo de histéresis que debe ser de 3-5 al final de este tiempo el contacto del relé debe cambiar desactivando la carga y apagando la luminaria.

Para presentar este tiempo de histéresis se hace una maquina de estados la cual tiene 4 estados los cuales son: encendido, apagado, calibración día y calibración noche, en la calibración día y calibración noche se hace el tiempo de histéresis y luego se hace una nueva lectura del sensor de luz para que pase al estado día o noche dependiendo de la lectura del sensor de luz.

\subsection{Generación y calibración del PWM}

El PWM se hace para controlar una salida la cual tiene un relé electromecánico con rangos de funcionamiento de 0 a $24 \mathrm{Vdc}$ cuando el contacto del relé está abierto y se controla a través de un transistor de alto voltaje al cual se le coloca al colector la señal sinusoidal rectificada, y a la base del transistor la salida del PWM del microcontrolador.

El microcontrolador tiene dos módulos TIMER que son TA0 y TA1 los cuales tienen un registro de control llamado TACTL, configurando este registro se pueden lograr varias configuraciones de funcionamiento ya sea como temporizador o contador.

Para generar el PWM se tienen que utilizar los contadores del TA0 y del TA1 del MSP430F2002 y activar el registro OUTMOD para poder gene- rar un periodo y un ciclo útil con los dos contadores, para el desarrollo se escogió enviar cuatro pulsos con modo PWM sobre la onda seno con para dar el voltaje al relé de 20 a $24 \mathrm{Vdc}$ y bajar el nivel de ruido que produce el relé en funcionamiento ecuación (6).

$$
\begin{aligned}
\text { Periodo } & =\frac{1}{8 \cdot 120[\mathrm{~Hz}]} \\
\text { Periodo } & =1041,6[\mu \mathrm{s}]
\end{aligned}
$$

La frecuencia de la onda rectificada presenta pequeñas variaciones que hacen que el PWM no siempre este sincronizado, para lo cual en la interrupción por puerto que detecta el cruce por cero de la señal rectificada, los registros de control TACTL y el OUTMOD siempre se borran y se vuelven a cargar para que el PWM siempre este sincronizado con la onda rectificada ecuación (7).

$$
\begin{gathered}
\frac{T A 1}{T A 0}=\text { Periodo } \cdot \text { Ciclo Util } \\
(\text { En hexadecimal })
\end{gathered}
$$

Para calibrar el PWM se utiliza el sensor de voltaje el cual detecta las variaciones de voltaje a la salida del rectificador. Para la calibración del PWM se hace una tabla de 20 valores el cual toma la posición de la salida del filtro de promedio móvil y mantiene el valor de PWM en un rango de $20 \mathrm{~V}$ a $24 \mathrm{Vdc}$ sobre el relé.

\subsection{Temporización}

La temporización se realiza cuando esta de noche y el contacto del relé está cerrada para que en algunas horas en la noche se apague la luminaria y baje el consumo de potencia, las horas son programadas según los requerimientos del usuario y para algunos sectores de la ciudad.

\subsection{Modo de ultrabajo consumo}

En la red de alumbrado público se presentan en ocasiones cortes de tensión, y estos cortes pueden ser nocturnos lo cual afecta el circuito que realiza la temporización al no tener tensión para su funcionamiento, por lo cual se le coloco un supercap de $0.22 \mathrm{~F}$ para que pueda man- 
tener la tensión en el circuito y realice la temporización aun cuando se presenten cortes de tensión en la red, el microcontrolador detecta automáticamente el corte de tensión en la red y apaga sus dos relojes principales y activa el reloj de ultra bajo consumo a $12 \mathrm{Khz}$ y activa la temporización en modo de ultra bajo consumo para que no se presenten fallas en la temporización.

El objetivo de colocar el modo de ultra bajo consumo es que el circuito tenga memoria en el momento de la temporización y se presente un corte en la tensión disponible sin que el microcontrolador se reseteé y pierda la temporización.

\section{RESULTADOS}

La figura 2 muestra el esquema eléctrico del fotocontrol.

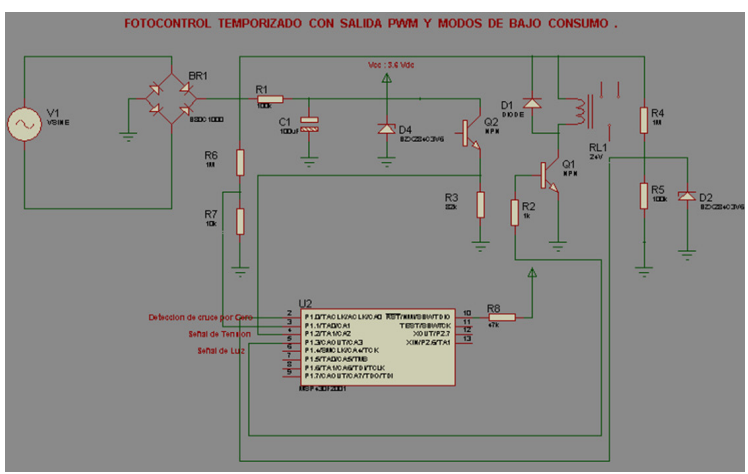

Figura 2. Esquema eléctrico del fotocontrol [13]

Se realizaron dos tipos pruebas de cada parte del fotocontrol para verificar que los resultados estén dentro de la norma NTC2470, la primera prueba se llama prueba de sensiblidad realizada sobre la celda fotosensible el cual es un fototransistor para verificar que el rango de encendido sea menor a 65 luxes y el rango de apagado este entre 10 y 22 luxes para lo cual para el circuito se ensayaron 3 resistencias para RE de diferentes valores.

El resultado de la sincronización se puede apreciar en la figura 3.

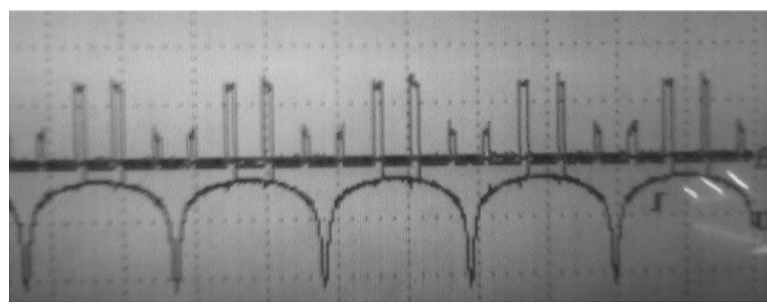

Figura 3. Imagen de sincronización de PWM con cruce por cero

La figura 2 muestra como se realizo el montaje con el fototransistor y por medio de un banco de prueba para fotocontroles en la empresa INADISA S.A, se ajusto la luminosidad del banco calibrado por un luxómetro a las escalas de la tabla 1, con diferentes resistencias, esos rangos son a los que el fotocontrol debe dar la orden de encender la luminaria o apagarla, se escogió la resistencia de $82 \mathrm{~K} \Omega$ porque los rangos de voltaje entre cada escala es mayor a $0,8 \mathrm{~V}$ para que la conmutación tenga una histéresis de aproximadamente 10 us.

Tabla 1. Voltaje de salida frente a una entrada de luz con tres diferentes resistencias

\begin{tabular}{|c|c|c|c|}
\hline Luxes/Resistencia & $\mathbf{8 2} \mathbf{K} \boldsymbol{\Omega}$ & $\mathbf{1 2 0 K} \boldsymbol{\Omega}$ & $\mathbf{1 8 0 K} \boldsymbol{\Omega}$ \\
\hline $\mathbf{6 5}$ & $2.84 \mathrm{~V}$ & $2.85 \mathrm{~V}$ & $2.87 \mathrm{~V}$ \\
\hline $\mathbf{2 0}$ & $1.75 \mathrm{~V}$ & $2.51 \mathrm{~V}$ & $2.79 \mathrm{~V}$ \\
\hline $\mathbf{1 0}$ & $0.87 \mathrm{~V}$ & $1.31 \mathrm{~V}$ & $1.70 \mathrm{~V}$ \\
\hline
\end{tabular}

Para el PWM se realizó la tabla 2 para ajustar el ciclo útil con un periodo de $1070 \mathrm{~Hz}$ para que se obtengan 4 pulsos en la onda seno de $60 \mathrm{~Hz}$ para obtener de una entrada de 105Vac a 305Vac en el circuito una entrada entre 20 y $24 \mathrm{Vdc}$ al relé.

En el microcontrolador MSP430F2002 se realizo la prueba para las frecuencias de $1041 \mathrm{~Hz}$, $1050 \mathrm{~Hz}, 1060 \mathrm{~Hz}$ y $1070 \mathrm{~Hz}$, para las frecuencias de $1041 \mathrm{~Hz}, 1050 \mathrm{~Hz}, 1060 \mathrm{~Hz}$ se presentaba ruido en la forma de onda variando el PWM, para $1070 \mathrm{~Hz}$ no se presentaba ruido sobre la forma de onda por lo cual se decide poner esta frecuencia como el periodo para el PWM y con base en este obtener los datos de la tabla 2 para el ciclo útil. 
Tabla 2. Resultados de Voltaje de entrada al circuito y ciclo útil del PWM a una frecuencia de $1041 \mathrm{~Hz}$

\begin{tabular}{|c|c|c|c|}
\hline Vac & Ciclo Util & Vac & Ciclo Util \\
\hline 105 & 820 & 210 & 945 \\
\hline 120 & 855 & 220 & 945 \\
\hline 130 & 870 & 230 & 950 \\
\hline 140 & 885 & 240 & 955 \\
\hline 150 & 900 & 250 & 960 \\
\hline 160 & 915 & 260 & 965 \\
\hline 170 & 920 & 270 & 965 \\
\hline 180 & 930 & 280 & 970 \\
\hline 190 & 935 & 290 & 975 \\
\hline 200 & 945 & 305 & 980 \\
\hline
\end{tabular}

El microcontrolador MSP430F2002 se encarga de leer el sensor de voltaje y hacer una lectura del valor de voltaje y dar un valor de la conversión en el ADC del microcontrolador, el valor a la salida del filtro de promedio móvil se muestra en la tabla 3.

Tabla 3. Valor del ADC filtrado el cual da el valor del ciclo útil del PWM

\begin{tabular}{|c|c|c|c|}
\hline ADC & Ciclo Útil & ADC & Ciclo Útil \\
\hline 0 & 820 & 7 & 945 \\
\hline 1 & 855 & 8 & 945 \\
\hline 2 & 870 & 8 & 950 \\
\hline 2 & 885 & 9 & 955 \\
\hline 3 & 900 & 9 & 960 \\
\hline 4 & 915 & 10 & 965 \\
\hline 5 & 920 & 10 & 965 \\
\hline 5 & 930 & 10 & 970 \\
\hline 6 & 935 & 11 & 975 \\
\hline 7 & 945 & 11 & 980 \\
\hline
\end{tabular}

Con esos datos se realiza la tabla 4 en el microcontrolador para la cual en cada valor del ADC filtrado se obtenga una valor de PWM y así dar un valor entre 20 y $24 \mathrm{Vdc}$ a la entrada de alimentación del relé y dar confiabilidad al conmutar la carga.

La figura 4 muestra tres formas de onda las cuales son la salida del PWM, la onda de entra- da a $60 \mathrm{~Hz}$ y la salida a $22 \mathrm{Vdc}$ sobre el relé.

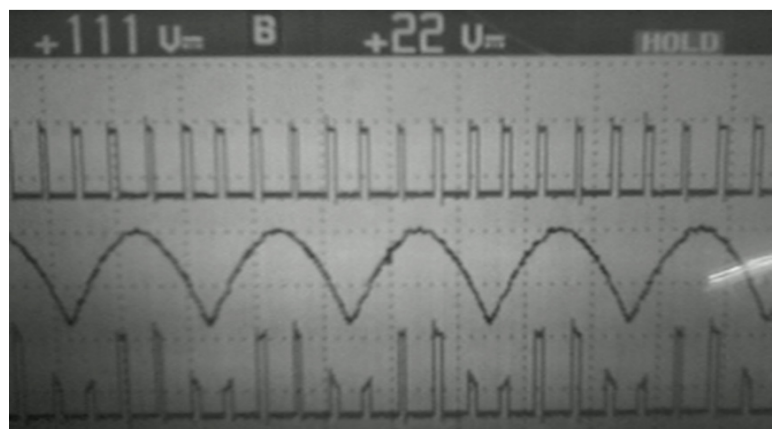

Figura 4. PWM sincronizado con la onda seno de entrada a $60 \mathrm{~Hz}$ a 111 Vac y salida sobre el relé a $22 \mathrm{Vdc}$

Para la temporización se obtuvieron los siguientes resultados teniendo una base de tiempo de dos horas de temporización con el microcontrolador MSP430F2002 con una calibración a $1 \mathrm{MHz}$ del reloj interno y se obtuvieron los resultados mostrados en la tabla 4.

Tabla 4. Resultados de la temporización realizada durante cinco días seguidos con el reloj interno del microcontrolador MSP430F2012

\begin{tabular}{|c|c|c|c|c|c|}
\hline & Lunes & Martes & Miércoles & Jueves & Viernes \\
\hline On & 1.58 .05 & 2.03 .08 & 2.05 .04 & 1.56 .38 & 2.01 .55 \\
\hline Off & 3.57 .34 & 4.02 .01 & 4.06 .25 & 3.58 .53 & 4.00 .34 \\
\hline
\end{tabular}

En el modo de ultrabajo consumo se realizo la prueba por cinco días seguidos apagando la fuente de alimentación y se obtuvieron los siguientes resultados consignados en la tabla 5 .

Tabla 5. Resultados del modo de ultrabajo consumo durante 5 días seguidos con el circuito sin alimentación de la red con temporización

\begin{tabular}{|c|c|c|c|c|}
\hline Lunes & Martes & Miércoles & Jueves & Viernes \\
\hline 4.15 .30 & 3.45 .23 & 4.05 .47 & 3.58 .13 & 4.07 .35 \\
\hline
\end{tabular}

Se observa que con el supercapacitor de $0.22 \mathrm{uF}$ (figura 5) se obtiene una duración bastante alta sin que el circuito se reseteé y pierda la temporización que lleva.

En los resultados obtenidos se observa que los valores teóricos se aproximan a los prácticos en el cálculo de la resistencia para los valores de 
funcionamiento pero para que sea más exacto toca calcular el porcentaje de error y sumarle ese porcentaje para que identifique exactamente cuando esta a 65 luxes y se encienda o cuando esté por debajo de 20 luxes y se apague teniendo un tiempo de histéresis de $3 \mathrm{~s}$ a $5 \mathrm{~s}$.

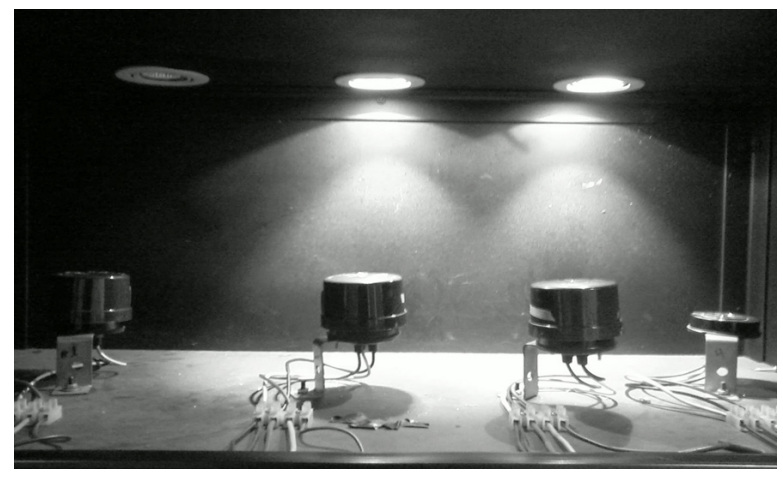

Figura 5. Prueba de contactos para los fotocontroles.

Para el PWM se obtiene según las ecuaciones (6) y (7) se obtuvieron valores diferentes debido a que en el trabajo realizado solo se tenía un solo pulso de cruce por cero, en este se implementaron cuatro pulsos con lo cual este resultado vario.

Para el correcto funcionamiento del PWM este debe estar sincronizado con la onda rectificada por lo cual se debe borrar el registro cada cruce por cero para garantizar el correcto funcionamiento en el PWM y que no se presenten desfases del PWM con respecto a la onda rectificada.

Se observo el reloj interno del MSP430F2002 en la temporización es muy preciso ya que el porcentaje de error es menor del $3 \%$.

En el modo de ultrabajo consumo en el microcontrolador MSP430F2002 se obtuvo una mayor confiabilidad al tener un mayor tiempo de temporización, al apagar la fuente debido a que se habían realizado pruebas con el MC68HC908QT2 de freescale pero temporiza un tiempo menor a 5 minutos el cual tenía una menor confiabilidad en la temporización al no tener tensión en la red.

\section{CONCLUSIONES}

En el presente trabajo se desarrolló un fotocontrol temporizado con modos de bajo consumo para la empresa INADISA S.A con un microcontrolador MSP430F2002 el cual se ajusta a las normas de consumo de potencia del fotocontrol temporizado, el cual trata las variables de tensión, luminosidad del ambiente, y sincronización de la onda de salida con la red a $60 \mathrm{~Hz}$.

El consumo de potencia del actual fotocontrol es alto al momento de activar la carga y la conmutación del contacto del relé no es confiable por lo cual con el nuevo diseño del fotocontrol se hace una temporización definida por el usuario para disminuir el consumo de potencia y por medio del sensor de luz con el filtro de promedio móvil la conmutación del contacto se hace más confiable al no presentarse transitorios de corriente elevadas que microsolden el contacto del relé.

Para realizar una correcta temporización se debe tener en cuenta los cortes de tensión que puedan afectar la temporización por lo cual se colocó un supercapacitor de $0.22 \mathrm{~F}$ y utilizar el microcontrolador un modo de ultrabajo consumo para una larga duración del supercapacitor $y$ tener en funcionamiento el circuito que realiza la temporización.

\section{RECONOCIMIENTOS}

A INADISA S.A empresa donde se desarrolló el fotocontrol temporizado con modos de bajo consumo. Al Ingeniero electrónico y especialista en automatización electrónica Ricardo Javier Buitrago González. Al Ingeniero Eléctrico y especialista en balastos Carlos David Solano Peña. 


\section{Referencias Bibliográficas}

[1] Norma técnica Colombiana NTC2470. [En línea], consultado en Octubre 30 de

RETILAP-FTT. [En línea], 2012, disponible en: http://www.scribd. com/doc/50132131/NTC24701994.

[2] Inadisa S.A, Codensa fotocontroles para HID. [En línea], consultado en Noviembre 11 de 2012, disponible en: http://www. inadisa.com/index.php/2013-04-18-0108-35/2013-04-18-03-13-12?id=119

[3] Ministerio de Minas y Energía, anexo general reglamento técnico de iluminación y alumbrado público. [En línea], consultado en Marzo 2 de 2013, disponible en: http://camacol.co/ sites/default/files/secciones_internas/ RETILAP_Resolucion_180540_del_30_ de_marzo_de_2010_0.pdf

[4] Imtran SRL, fotocontrol IMTRAN. [En línea], consultado en Diciembre 15 de 2012, disponible en: http://www.imtran. com.uy/web/?page_id=228

[5] Inadisa S.A, fotocontrol electrónico Azul. [En línea], consultado en Enero 22 del 2013, disponible en: http://www. inadisa.com/Fotocontrol $\% 20$ AZUL $\% 20$ $120-277$ V\%20N.C\%20Fail\%20On\%20 ref\%203NC16A.pdf

[6] Inadisa S.A, fotocontrol electrónico. [En línea], consultado en Septiembre 18 de 2012, disponible en: http://www.inadisa. com/index.php/2013-04-18-01-0835/2013-04-18-03-13-12 disponible en: http://www.minminas. gov.co/minminas/downloads/ archivosSoporteRevistas/7853.pdf.

[8] D. Gutiérrez; Principales aspectos de la iluminación en contexto con la reglamentación técnica. [En línea], consultado en Octubre 9 de 2012, disponible en: http://www. fenalcoantioquia.com/res/itemsTexto/ recursos/cap_retilap.pdf

[9] E. Afanador; ASOCODIS Y ANDESCO, 2009. [En línea], consultado en Septiembre 10 de 2012, disponible en: http://www.asocodis.org.co/cms/Default. asp? Page $=168$

[10] J. Davies; MSP430 Microcontroller basics, Editorial Kindle, pp 550-555, 2011

[11] Texas Instruments, MSP430x20x1, MSP430x20x2, MSP430x20x3 Mixed signal microcontroller, 2007. [En línea], consultado en Octubre 7 de 2012, disponible en: http://akizukidenshi.com/ download/MSP430F2013IN.pdf

[12] B. Nisarga; PWM DAC using MSP430 high-resolution timer, pp. 1-26, 2011.

[13] R. Buitrago; Diseño de un modelo de telegestión para monitoreo de luminarias de alumbrado público, Tesis de grado, Universidad de la Salle, 2011. 